\title{
Towards laser spectroscopy of pionic helium atoms at Paul Scherrer Institute
}

\author{
Masaki Hori ${ }^{* a}$ Anna Sótér, ${ }^{a}$ Hossein Aghai-Khozani, ${ }^{a}$ Daniel Barna, ${ }^{b c}$ Andreas Dax, ${ }^{d}$ \\ Ryugo S. Hayano, ${ }^{e}$ Yohei Murakami, ${ }^{e}$ Koichi Todoroki, ${ }^{e}$ and Hiroyuki Yamada ${ }^{e}$ \\ ${ }^{a}$ Max-Planck-Institut für Quantenoptik, D-85748 Garching, Germany \\ ${ }^{b}$ CERN CH-1211 Geneva 23, Switzerland \\ ${ }^{c}$ Wigner Research Center for Physics, H-1525 Budapest, Hungary \\ ${ }^{d}$ Paul Scherrer Institut, $\mathrm{CH}-5232$ Villigen, Switzerland \\ ${ }^{e}$ Department of Physics, School of Science, University of Tokyo, Tokyo 113-0033, Japan
}

\begin{abstract}
Metastable pionic helium is a hypothetical three-body atom composed of a helium nucleus, a $\pi^{-}$ occupying a Rydberg state, and an electron occupying the ground state. The PiHe collaboration is currently attempting to carry out laser spectroscopy of these atoms using the $590-\mathrm{MeV}$ ring cyclotron of the Paul Scherrer Institute. We briefly describe the method by which we intend to detect the spectroscopic signal.
\end{abstract}

Technology and Instrumentation in Particle Physics 2014,

2-6 June, 2014

Amsterdam, the Netherlands

${ }^{*}$ Speaker. 


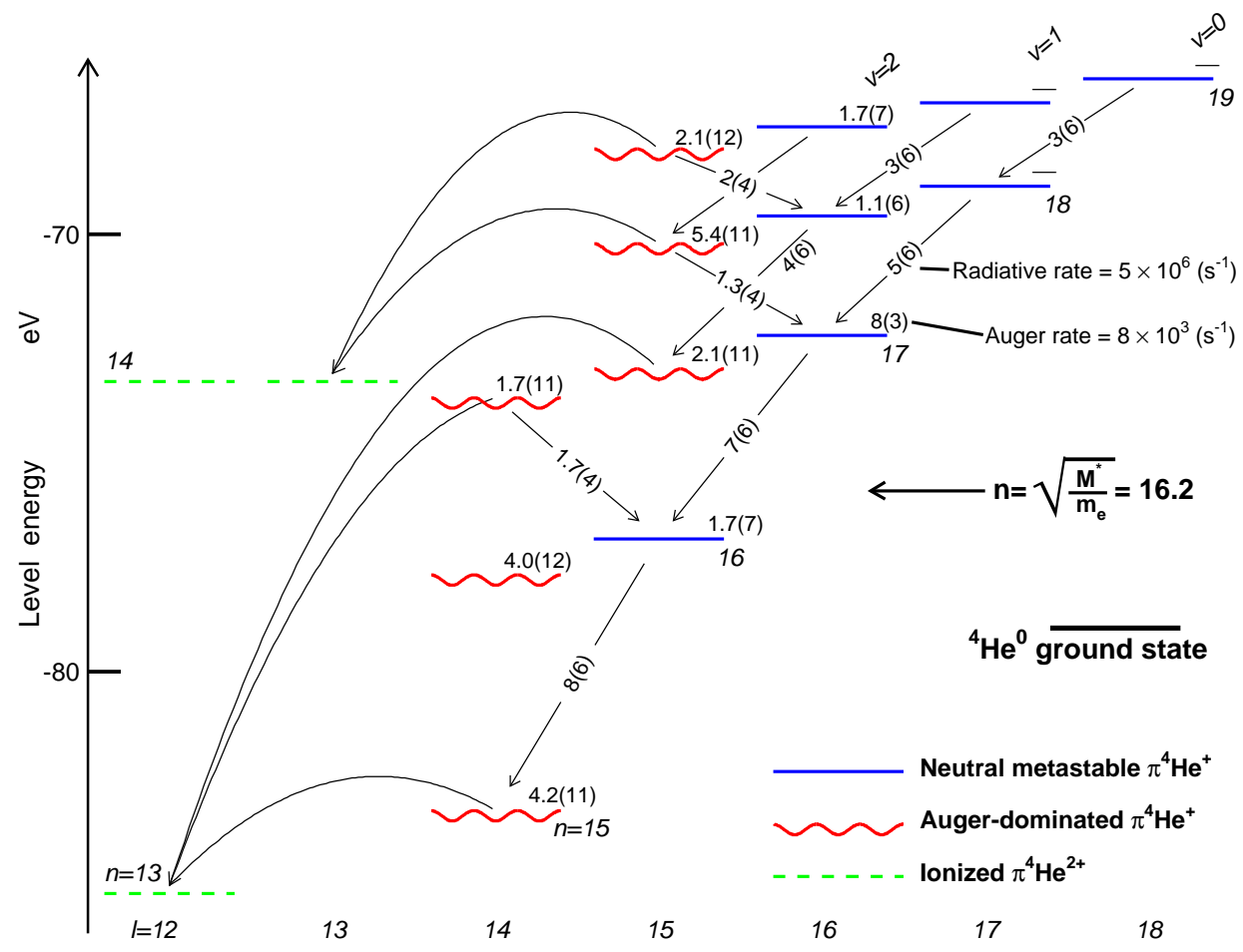

Figure 1: Energy level diagram of $\pi^{4} \mathrm{He}^{+}$atom. On the left scale the theoretical absolute energy of each state $(n, \ell)$ is plotted relative to the three-body-breakup threshold. The wavy lines indicate states with picosecond-scale lifetimes, solid lines the metastable levels with lifetimes of $>10 \mathrm{~ns}$. Strong radiative transitions of the types $(n, \ell) \rightarrow(n-1, \ell-1)$ and $(n, \ell) \rightarrow(n-1, \ell+1)$ involving the emission of a UV or visible photon are indicated using straight arrows, with the corresponding decay rates shown in $\mathrm{s}^{-1}$. The decay rate of Auger emission of the $1 s$ electron for each state is shown in $\mathrm{s}^{-1}$. The curved arrows indicate some dominant Auger transitions. The dashed lines indicate the two-body $\pi \mathrm{He}^{2+}$ ionic states formed after Auger emission. Figure from Ref. [四].

\section{Introduction}

The PiHe collaboration of PSI is currently attempting to carry out laser spectroscopy [四] of metastable pionic helium $\left(\pi \mathrm{He}^{+} \equiv \pi^{-}+\mathrm{He}^{2+}+e^{-}\right)$. This is a heretofore hypothetical three-body atom [ [ 2 , [3] composed of a helium nucleus, an electron in the $1 \mathrm{~s}$ ground state, and a $\pi^{-}$in a Rydberg state with principal and orbital angular momentum quantum numbers of around $n \sim \ell+1 \sim 16$. The atoms are formed by the process

$$
\pi^{-}+\mathrm{He} \rightarrow \pi \mathrm{He}^{+}+e^{-}
$$

that spontaneously occurs when a beam of $\pi^{-}$is allowed to come to rest in a liquid helium target. These Rydberg states are expected to retain nanosecond-scale lifetimes against $\pi^{-}$nuclear absorption and $\pi^{-} \rightarrow \mu^{-}+\bar{v}_{\mu}$ decay. This is because the Rydberg $\pi^{-}$orbitals have very little overlap with the nucleus, whereas the electromagnetic cascade processes that normally lead to immediate nuclear absorption are suppressed. A laser spectroscopic signal would conclusively show the existence of $\pi \mathrm{He}^{+}$. By comparing the experimental frequencies with the results of the three-body QED 
calculations, the $\pi^{-}$mass can be determined with a high precision, as in the case of antiprotonic helium $\left(\bar{p} \mathrm{He}^{+}\right)$atoms [困, [1, 目, 口, [8].

The existence of $\pi \mathrm{He}^{+}$has been inferred from several experiments carried out in the 1960's

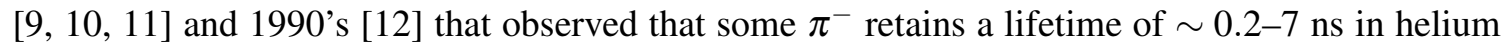
targets. Nothing is experimentally known about the $n$ and $\ell$ distribution of states which may be formed, and no atomic lines of $\pi \mathrm{He}^{+}$have been detected so far. The lifetimes of some $\pi \mathrm{He}^{+}$states calculated recently [四] using the complex-coordinate rotation method appear to differ by 1-2 orders of magnitude from earlier estimations made in the 1960's.

Several assumptions are needed to design the laser spectroscopy experiment. The principal quantum number of the initially-populated states is assumed to be distributed around the value

$$
n \sim n_{0}=\sqrt{\frac{M^{*}}{m_{e}}} \sim 16
$$

Here $m_{e}$ and $M^{*}$ respectively denote the electron mass and the reduced mass of the $\pi^{-}-\mathrm{He}^{2+}$ system. This corresponds to a $\pi^{-}$orbital with the same radius and binding energy as those of the displaced $1 s$ electron. In the case of $\bar{p} \mathrm{He}^{+}$, laser spectroscopy experiments [13], [4]] support the simple estimation of Eq. [.2. The calculations of Ref. [W] indicate that the Auger decay rate of state $(n, \ell)=(16,15)$ is relatively small $\left(1.7 \times 10^{7} \mathrm{~s}^{-1}\right.$, see Fig. 四).

Several transitions can in principle be studied, but we selected $(n, \ell)=(16,15) \rightarrow(17,14)$ in the $\pi^{4} \mathrm{He}^{+}$isotope for our initial attempts at laser spectroscopy. Three-body calculations that include relativistic and quantum electrodynamics corrections of orders $R_{\infty} \alpha^{2}$ and $R_{\infty} \alpha^{3}$ in atomic units, where $R_{\infty}$ and $\alpha$ denote the Rydberg and fine structure constants, predict a transition frequency of $v_{\text {th }} \sim 781053 \mathrm{GHz}$ [四] for this transition.

\section{Laser spectroscopy method}

We propose the irradiation of the $\pi \mathrm{He}^{+}$with laser pulses that induce transitions from the metastable state $(16,15)$, to a state $(17,14)$ with a picosecond-scale lifetime against Auger emission of the $1 s$ electron (Fig. W). The Rydberg $\pi \mathrm{He}^{2+}$ ion that remains after Auger decay undergoes Stark mixing with $s, p$, and $d$ states (see Ref. [W] and references therein) during collisions with the helium atoms in the experimental target. As these low- $\ell$ states have a large overlap with the helium nucleus, the $\pi^{-}$undergoes nuclear absorption within picoseconds. Neutrons, protons, deuterons, and tritons with kinetic energies of up to $E=30-90 \mathrm{MeV}$ emerge. By measuring these particle rates, the resonance condition between the laser and the $\pi \mathrm{He}^{+}$is revealed in the form of a resonance curve.

In Fig. 凤, the resonance profile of the $\pi^{4} \mathrm{He}^{+}$transition $(n, \ell)=(16,15) \rightarrow(17,14)$ excited with a 1-ns-long laser pulse of fluence $I \sim 6 \mathrm{~mJ} \mathrm{~cm}^{-2}$ is simulated. The intensity of the $\pi^{-}$absorption spike is plotted as a function of the laser frequency $\Delta v$ detuned from the resonance frequncy $v_{\text {exp }}$. The width of this profile is predominantly caused by the Auger decay rate of the resonance daughter state $(17,14)$, and the $14-\mathrm{GHz}$ spacing between the fine structure sublines that arise from the interaction between the electron spin and the orbital angular momentum of the $\pi^{-}$. Simulations [U] indicate that the centroid of this profile can in principle be determined with a precision of $<1$ $\mathrm{GHz}$, which corresponds to a fractional precision of better than $\sim 1 \times 10^{-6}$ on the $\pi \mathrm{He}^{+}$transition frequency $v_{\text {exp }}$. Other laser transitions, e.g., $(17,16) \rightarrow(16,15)$ between metastable states are predicted to have resonance lineshapes that are much narrower. 


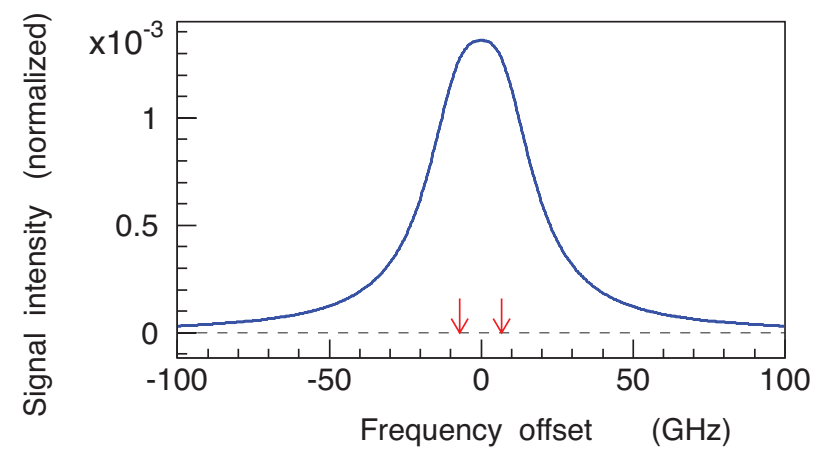

Figure 2: Simulated profile of the laser resonance of the $\pi^{4} \mathrm{He}^{+}$transition $(n, \ell)=(16,15) \rightarrow(17,14)$. Arrows indicate the positions of the dominant fine structure sublines (see text). Figure from Ref. [四].

The $\pi \mathrm{He}^{+}$are synthesized by allowing a $85-\mathrm{MeV} \pi^{-}$beam of intensity $>10^{7} \mathrm{~s}^{-1}$ produced by the PSI ring cyclotron [155, [6] to come to rest in the liquid helium target. The precise time structure of this $\pi^{-}$beam allows the formation of $\pi \mathrm{He}^{+}$to be synchronized to the arrival of the resonant laser pulses at the target [四]. The 1-ns-long laser pulses of wavelength $\lambda=383.8 \mathrm{~nm}$ will be generated by a frequency-doubled hybrid dye/Ti:Sapphire laser which is pumped by a diodepumped solid-state (DPSS) Nd:YAG laser. The typical energy and repetition rate of the laser is $\sim 10 \mathrm{~mJ}$ and $f_{r}>100 \mathrm{~Hz}$. The protons, neutrons, deuterons, and tritons which emerge from the experimental target are detected by an array of 40-mm-thick scintillation counters [四. Extruded plastic scintillation counters [[1]] may also be used. Monte-Carlo simulations indicate that a $\pi \mathrm{He}^{+}$ resonance can be detected in a few weeks of beamtime. The PiHe collaboration is scheduled to carry out the first physics beamtime in August 2014.

\section{Outlook}

The $\pi^{-}$mass has been previously determined to a fractional precision of $10^{-5}-10^{-6}$ in two ways, i): the x-ray transition energies of some $\pi^{-}$atoms were compared with relativistic boundstate calculations [18], [Q]], or ii): the recoil momentum [20] of the $\mu^{+}$which emerged from a stationary $\pi^{+}$undergoing decay was precisely measured. Depending on the atomic cascade models used in the analysis of the $\mathrm{x}$-ray transition energies, the $\pi^{-}$mass values varied by $\sim 10^{-5}$ [ए8]].

We intend to determine the $\pi^{-}$mass by comparing the measured frequencies of $\pi \mathrm{He}^{+}$with the results of the three-body QED calculations described above. The precision of this determination will be ultimately limited by the natural width of the $\pi \mathrm{He}^{+}$resonance as determined by the lifetime $\tau_{\pi} \sim 26 \mathrm{~ns}$ of $\pi^{-}$, compared to the transition energy $v_{\exp }$ [回]. A fractional precision on the $\pi^{-}$mass of $<10^{-8}$ can in principle be achieved, as in the case of $\bar{p} \mathrm{He}^{+}$[四, 四]. In practice, systematic effects such as the shift and broadening of the resonance line due to atomic collisions in the target, AC Stark shifts [2]], spurious frequency chirp in the pulsed laser beam [22], and statistical uncertainties can prevent the experiment from achieving this precision. The lifetimes of some $\pi \mathrm{He}^{+}$states may be shorter than $\tau_{\pi}$, due to collisions with helium atoms in the experimental target [[23]]. 


\section{Acknowledgments}

We are deeply indebted to the PSI particle physics and beamline groups for their support. We thank V.I. Korobov for theoretical discussions. This work was supported by the European Research Council (ERC-StG).

\section{References}

[1] M. Hori, A. Sótér, and V.I. Korobov, Phys. Rev. A 89, 042515 (2014).

[2] G.T. Condo, Phys. Lett. 9, 65 (1964).

[3] J.E. Russell, Phys. Rev. Lett. 23, 63 (1969).

[4] M. Hori et al., Nature 475, 484 (2011).

[5] V.I. Korobov, Phys. Rev. A 77, 042506 (2008).

[6] V.I. Korobov, L. Hilico, and J.-P. Karr, Phys. Rev. Lett. 112, 103003 (2014).

[7] M. Hori et al., Phys. Rev. Lett. 96, 243401 (2006).

[8] M. Hori et al., Phys. Rev. Lett. 91, 123401 (2003).

[9] J.G. Fetkovich and E.G. Pewitt, Phys. Rev. Lett. 11, 290 (1963).

[10] M.M. Bloch et al., Phys. Rev. Lett. 11, 301 (1963).

[11] O.A. Ză̈midoroga, R.M. Sulyaev, and V.M. Tsupko-Sitnikov, Sov. Phys. JETP 25, 63 (1967).

[12] S.N. Nakamura et al., Phys. Rev. A 45, 6202 (1992).

[13] M. Hori et al., Phys. Rev. Lett. 89, 093401 (2002).

[14] T. Kobayashi et al., J. Phys B: At. Mol. Opt. Phys. 46, 245004 (2013).

[15] W. Wagner et al., Nucl. Instrum. Methods Phys. Res. Sect. A 600, 5 (2009).

[16] J. Adam et al., Eur. Phys. J. C 73, 2365 (2013).

[17] A. Sótér, K. Todoroki, T. Kobayashi, D. Barna, D. Horváth, and M. Hori, Rev. Sci. Instrum. 85, 023302 (2014).

[18] B. Jeckelmann, P. F. A. Goudsmit, and H. J. Leisi, Phys. Lett. B 335, 326 (1994).

[19] S. Lenz et al., Phys. Lett. B 416, 50 (1998).

[20] K. Assamagan et al., Phys. Rev. D 53, 6065 (1996).

[21] M. Hori and V.I. Korobov, Phys. Rev. A 81, 062508 (2010).

[22] M. Hori and A. Dax, Opt. Lett. 34, 1273 (2009).

[23] M. Hori et al., Phys. Rev. A 70, 012504 (2004). 